\title{
Blastic Plasmacytoid Dendritic Cell Neoplasm: A Rare Case Report with Literature Review
}

\author{
Nikita Oza ${ }^{1, *}$ Krishnakumar Rathnam ${ }^{2, *}$ Gujral Sumeet ${ }^{3}$ Susan R. Honey ${ }^{2}$ S. V. Saju ${ }^{20}$ \\ Kane Shubhada ${ }^{1}$ \\ 1 Department of Histopathology, SRL Diagnostics - Centre of \\ Excellence, Goregaon West, Mumbai, Maharashtra, India \\ ${ }^{2}$ Department of Medical Oncology, Meenakshi Mission Hospital \& \\ Research Centre, Madurai, Tamil Nadu, India \\ ${ }^{3}$ Department of Pathology, Tata Memorial Hospital, Parel, Mumbai, \\ Maharashtra, India \\ Ind J Med Paediatr Oncol 2021;42:496-500. \\ Address for correspondence Nikita Oza, MD, FIAC, Department of \\ Histopathology, SRL Diagnostics - Centre of Excellence, S.V. Road, \\ Goregaon West, Mumbai, 400016, Maharashtra, India \\ (e-mail:drnikitaoza@gmail.com).
}

\author{
Abstract \\ Keywords \\ - blastic plasmacytoid \\ dendritic cell \\ neoplasm \\ - chemotherapy \\ - stem cell therapy \\ - targeted therapy
}

\section{Introduction}

Blastic plasmacytoid dendritic cell neoplasm (BPDCN) is a rare and aggressive hematologic malignancy with an incidence of $0.000045 \%$ and constitutes less than $1 \%$ of all acute leukemia. ${ }^{1}$ This neoplasm predominantly affects elderly males with the median age being in the sixth decade of life and sex ratio of 3:1.,3 It remained an orphan disease for long until recently when few series have been published with predominant cutaneous involvement making it difficult to

\footnotetext{
Authors have equally contributed for writing, review and editing the case report.
}

establish clinical, biological, and prognostic features. ${ }^{4}$ We present this rare case and discuss the diagnostic approach with various treatment options available for this entity.

\section{Case Report}

A 70-year-old male patient presented with insidious onset of multiple reddish painless nodules all over the body for the last 3 months. The nodules were of varying sizes ranging from 0.5 to $2 \mathrm{~cm}$ in diameter. The lesions started in the chest region, later spread over forearm, arm, thighs and then all over the body. Nodules were nonpruritic, nontender, and nonpedunculated.

\footnotetext{
(c) 2021. Indian Society of Medical and Paediatric Oncology. All rights reserved.

This is an open access article published by Thieme under the terms of the Creative Commons Attribution-NonDerivative-NonCommercial-License, permitting copying and reproduction so long as the original work is given appropriate credit. Contents may not be used for commercial purposes, or adapted, remixed, transformed or built upon. (https://creativecommons.org/ licenses/by-nc-nd/4.0/)

Thieme Medical and Scientific Publishers Pvt. Ltd., A-12, 2nd Floor, Sector 2, Noida-201301 UP, India
} 


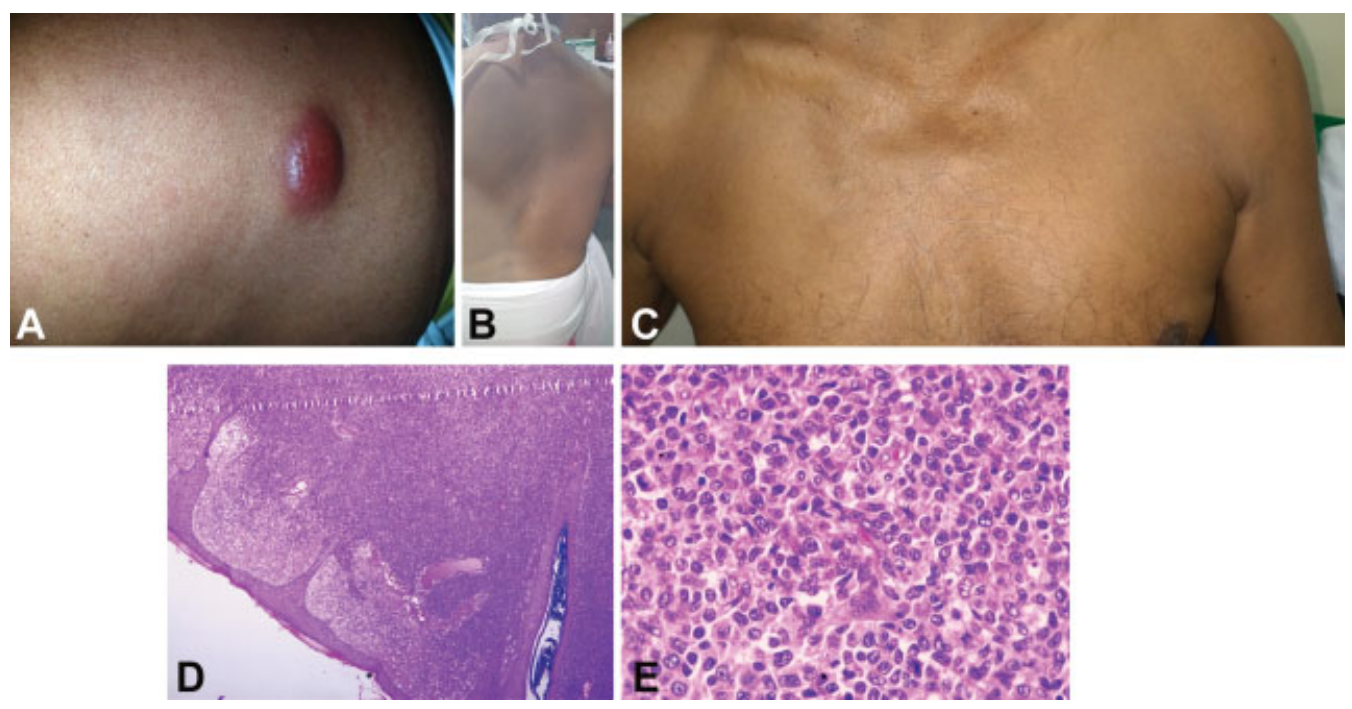

Fig. 1 (A) Reddish painless nodules over the trunk; (B, C) Complete disappearance of all nodules by second week of therapy; (D, E) Hematoxylin and eosin-stained section show dermal tumor. The tumor cells are intermediate in size and show oval irregular nuclear contour, conspicuous nucleoli, and moderate cytoplasm. Brisk mitotic figures and apoptotic bodies noted (original magnification: $1 \mathrm{~d}-\times 200$ and $1 \mathrm{e}-\times 400$ ).

Clinically, no palpable lymphadenopathy or organomegaly was noted. The Eastern Cooperative Oncology Group (ECOG)/ Karnofsky's index of performance status (KPS) performance score was 1. Hemogram was within normal limits with hemoglobin of $13 \mathrm{~g} / \mathrm{dL}$, white blood cell count of $8,400 / \mu \mathrm{L}$, and platelets of $380 \times 10^{9} / \mathrm{L}$. Contrast-enhanced scan of chest, abdomen, and pelvis was performed and revealed no significant abnormality. The lesions were not associated with type B symptoms or bleeding manifestations ( - Fig. 1A). In the given clinicoradiological context, possibility of a soft tissue sarcoma and cutaneous lymphoma was considered.

Complete excision biopsy of the nodule over the arm was done, and the blocks were processed and studied for histomorphology. Biopsy from the nodule revealed dermis infiltrated by atypical lymphoid cells arranged in diffuse sheets. Periadnexal and perivascular neoplastic cell aggregates were noted. The tumor cells were of intermediate size and showed oval nuclei, irregular nuclear contour, conspicuous nucleoli, and moderate eosinophilic cytoplasm. Prominent proliferating blood vessels were noted in the background. Frequent mitotic figures, atypical mitosis, and apoptotic bodies were identified. The tumor infiltrated the underlying subcutaneous tissue. Overlying epidermis was free of tumor ( - Fig. 1D and E).

On performing immunohistochemistry, the tumor diffusely expressed LCA (leukocyte common antigen) HLA DR (human leukocyte antigen - DR isotype), CD123, CD56, CD123, CD43, CD4, and CD68. Tumor cells were immunonegative for T-cell markers (CD3, CD8, and CD7), myeloid and monocytic cells (MPO, and CD117), B-cells (CD20), CK, CD10, BCL6, CD23, TIA, CD34, TdT, MUM1, TCR gamma and TCR Beta and EBV. TCL1 immunomarker was not performed due to unavailability. The MIB 1 labeling index was approximately 30\% in areas of highest proliferative activity ( - Fig. 2A-F). Bone marrow biopsy did not reveal infiltration by abnormal cells. Thus, diagnosis of blastic plasmacytoid dendritic cell neoplasm (BPDCN) was made.

Considering the age of the patient, allogenic stem cell transplant (allo-stem cell transplantation [SCT]) was at consoli- dation. The patient was then initiated on a modified BerlinFrankfurt-Munster (BFM) acute lymphoblastic leukemia (ALL)like protocol with prednisolone, $60 \mathrm{mg} / \mathrm{m}^{2}$; weekly vincristine, $1.4 \mathrm{mg} / \mathrm{m}^{2}$; daunorubicin, $25 \mathrm{mg} / \mathrm{m}^{2}$; and L-asparaginase, 5,000 unit $/ \mathrm{m}^{2}$ ( 2 doses a week as per institutional protocol adjusted to his age; total of 8 doses were delivered). He demonstrated a good response with complete disappearance of all nodules by second week of therapy (-Fig. 1B and C) and is continuing the same modified BFM ALL-like protocol. The patient is being closely monitored and did not have any side effects like hyperglycemia, pancreatitis, or bleeding manifestation. Clinically, patient went into remission after third week induction with no new lesions and disappearance of nodules.

\section{Discussion}

BPDCN is a rare malignancy of dendritic cell precursors as it resembles the normal counterpart in expression profile, immunophenotype, and biological function. ${ }^{5,6} \mathrm{~A}$ study of its ontogeny shows the cell of origin to be much closer to myeloid precursors. ${ }^{7}$ These are commonly called as natural killer (NK)cell lymphoma, CD4+NK-cell leukemia, or blastic NK leukemia/lymphoma due to the expression of the CD56 molecule. The etiology of BPDCN is not clearly known except for its association with myelodysplastic syndromes (MDS) due to the cooccurrence of several gene mutations. 6,7

The disease commonly presents as asymptomatic cutaneous lesions. ${ }^{7,8}$ Extracutaneous involvement includes involvement of bone marrow and lymph nodes. ${ }^{7}$ An isolated or disseminated bruise-like lesion has better outcome than more disseminated lesions. ${ }^{9-12}$ However, lack of initial cutaneous involvement has been reported in the literature like mucosal lesion in oral cavity. ${ }^{13}$

In cases of disseminated disease; biopsy of the cutaneous nodule, lymph node, or bone marrow biopsy is essential for establishment of the diagnosis. In cutaneous lesions, epidermis is usually spared, and the lesion typically infiltrates the 

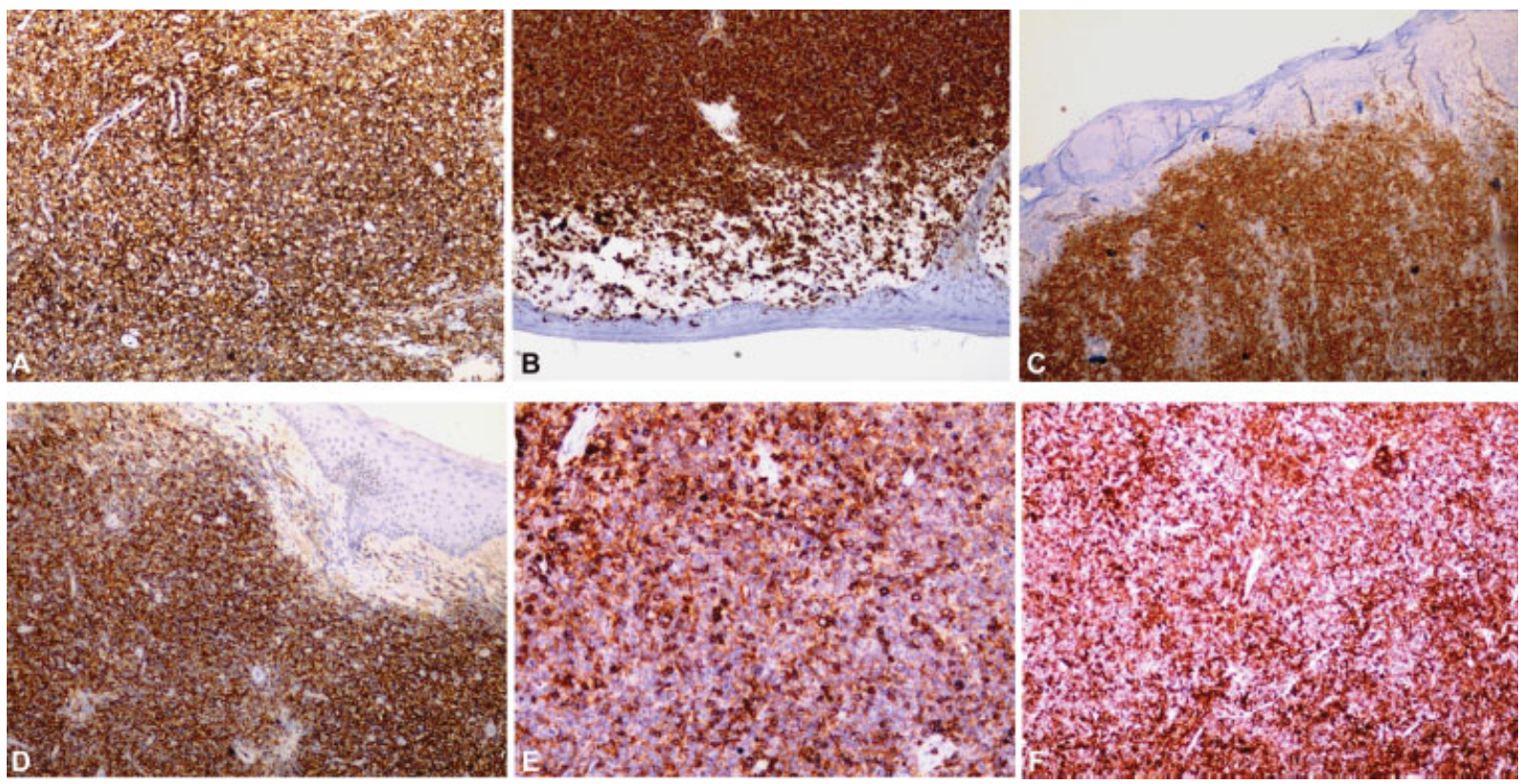

Fig. 2 The tumor cells expresses LCA (A), HLA-DR (B), CD123 (C), CD56 (D), CD43 (E), CD 68 (F) (original magnification: $\times 200$ ).

dermis. As seen in our case, progression of disease often leads to involvement of subcutaneous fat. Monomorphic population of small to medium sized cells showing irregular nuclear contours, fine to evenly dispersed chromatin, small nucleoli, and scant to moderate amount of cytoplasm is characteristically seen in BPDCN. ${ }^{14-17}$ Plasmacytoid dendritic cells (PDCs) showed strong immunoexpression for CD4, CD123, and HLADR, while they focally show CD43 and CD68. Expression of CD123 (high) is the main characteristic of PDCs. When in doubt, immunomarker for TCL1 may be used to support the diagnosis of BPDCN. Plasmacytoid dendritic cells are the principal source of interferon $\alpha$, and their accumulation at inflammatory sites contributes to the immune and inflammatory response. ${ }^{14,15}$

Plasmacytoid dendritic cells are predominantly seen in allergic rhinitis, hyaline vascular Castleman's disease, granulomatous lymphadenitis, Kikuchi-Fujimoto lymphadenopathy, and Hodgkin lymphoma. PDCs are also noticed in skin of patients with hydroa vacciniforme, lupus erythematosus, and psoriasis. ${ }^{14,15}$ In our case, the patient did not complain of any inflammatory lesion in the past. There is a strong debate that PDCs belong to the myelomonocytic lineage as AML - Acute Myeloid Leukemia and myelodysplasia can occur in the due course of this disease. World Health Organization Classification of Tumors of Hematopoietic and Lymphoid Tissue (2008) had grouped BPDCN with the category of AML and related precursor neoplasms which now has been listed as its own category in the 2016 revised version. ${ }^{18,19}$

BPDCN demonstrates deletion of several tumor suppressor genes including CDKN2A, RB1, CDKN1B and tumor protein p53 (TP53). TET2 gene mutation (ten-eleven translocation2 ), located on band $4 q 24$, provides additional evidence that BPDCN is a myeloid related neoplasm. ${ }^{16}$

A biological hallmark of BPDCN is disruption at the G1/S transition that possibly contributes to pathogenesis. ${ }^{16}$ Clini- cal features, morphologic findings, immunophenotypic profile, and cytogenetic and molecular data forms the basis for the diagnosis of BPDCN. ${ }^{16-18}$ Among these, immunoprofile of neoplastic cells plays an important role in the diagnosis of the disease since there is an overlap of the immunophenotypic features with other hematopoietic neoplasms. ${ }^{19}$

Before establishing a diagnosis of BPDCN in particular, NK-cell lymphoma/leukemia, mature T-cell lymphomas/ leukemias, T-cell lymphoblastic leukemia/lymphoma (T-ALL/LBL), and myeloid sarcoma/AML some should be excluded. ${ }^{17}$ In our case, myeloid sarcoma was ruled out by immunonegativity of MPO and CD117; T-ALL/LBL was ruled out as the tumor cells were immunonegative for T-cell markers (CD3, CD8, and CD7); NK T-cell lymphoma/leukemia was ruled out as the tumor cells did not express T-cell nor cytotoxic markers.

BPDCN is inherently resistant to standard chemotherapies and have overall poor survival. Intensive ALL-like induction regimens (e.g., cyclophosphamide, vincristine, doxorubicin [also known by its trade name, Adriamycin], and dexamethasone hyper-CVAD - cyclophosphamide, vincristine, doxorubicin (also known by its trade name, Adriamycin), and dexamethasone; BFM chemotherapy) a were considered as more effective on comparison to standard therapies like CHOP - cyclophosphamide, doxorubicin hydrochloride (hydroxydaunorubicin), vincristine sulfate (Oncovin), and prednisone. ${ }^{4,20,21}$

Combination of L-asparaginase and methotrexate has shown clinical activity in BPDCN, predominantly in elderly patients. $^{22,23}$ Consolidation with allogeneic SCT (allo-SCT), in first CR - Complete response, demonstrated durable complete remissions with overall survival rates ranging from 58\% at 3 years to $40 \%$ at 10 years. With regard to relapse rates, reduced intensity conditioning appears to be equivalent to myeloablative regimens. ${ }^{24}$ Elderly patients are treated with lower intensity chemotherapy regimens like pralatrexate, bendamustine, or 
gemcitabine/docetaxel combinations or hypomethylating agents like 5-azacytidine which demonstrated transient responses. $^{25-28}$

\section{Novel Agents in Blastic Plasmacytoid Dendritic Cell Neoplasm}

Literature reveals that low-intensity treatments have unsatisfactory results, whereas intensive therapies lead to toxicity. Thus, there was a need for the use of novel targeted agents for the treatment of BPDCN. ${ }^{29}$ SL-401 is a novel recombinant protein which includes components of diphtheria toxin fused with interleukin-3, demonstrating overall response rate of $77 \%$ (with 55\% CR). ${ }^{29}$ Promising result with CR rate of $79 \%$ (first line) and 31\% CR rate, a phase-II study with SL401 has been shown and reported at the 2017 ASH - American Society of Hematology for relapsed/refractory patients. ${ }^{27-29}$ Phase-I trials are ongoing with other immunotherapies targeting CD123 which includes immunoconjugates, chimeric antigen receptor (CAR)T-cells, and bispecific antibodies. ${ }^{28}$

Venetoclax (BCL-2 inhibitor) has demonstrated high single-agent activity in myeloid malignancies. Currently, it is used in combination with induction chemotherapy. Bromodomain and extra terminal domain inhibitors (BETs) are being studied in preclinical tests. ${ }^{27,28}$

SCT with myeloablative or reduced intensity regimen have shown improved survival in younger and selected elderly patients. The clinical course and response to the treatment is best when the procedure is performed after first CR. ${ }^{28-30}$

\section{Conclusion}

BPDCN is a very rare neoplasm with poor treatment outcomes. In younger patients, induction regimens (ALL-like) followed by allogenic SCT in CR-1 and in elderly unfit patients, L-asparaginase based chemotherapy or hypomethylating agents achieve good palliation. Novel therapies like anti-CD123-directed immunotherapies and venetoclaxbased therapy have shown promise in clinical trials.

\section{Ethics Statement}

The authors hereby declare that Institutional Ethics Committee did not mandate IEC review for this study. The collection of data from electronic medical record (EMR) and laboratory reports do not require an informed consent form as per the hospital's standard operating procedures.

\section{Conflict of Interest}

The authors declare that there is no conflict of interest.

\section{References}

1 Sapienza MR, Pileri A, Derenzini E, et al. Blastic plasmacytoid dendritic cell neoplasm: state of the art and prospects. Cancers (Basel) 2019;11(05):595

2 Facchetti F, Pileri SA, Agostinelli C, et al. Cytoplasmic nucleophosmin is not detected in blastic plasmacytoid dendritic cell neoplasm. Haematologica 2009;94(02):285-288
3 Garnache-Ottou F, Feuillard J, Saas P. Plasmacytoid dendritic cell leukaemia/lymphoma: towards a well defined entity? Br J Haematol 2007;136(04):539-548

4 Pagano L, Valentini CG, Pulsoni A, et al; GIMEMA-ALWP (Gruppo Italiano Malattie EMatologiche dell'Adulto, Acute Leukemia Working Party) Blastic plasmacytoid dendritic cell neoplasm with leukemic presentation: an Italian multicenter study. Haematologica 2013;98(02):239-246

5 Swerdlow SH, Campo E, Harris NLet al, eds. WHO Classification of Tumours of Haematopoietic and Lymphoid Tissues. WHO Classification of Tumours. Revised 4th ed. Vol 2. Lyon, France: International Agency for Research on Cancer; 2017

6 Sapienza MR, Abate F, Melle F, et al. Blastic plasmacytoid dendritic cell neoplasm: genomics mark epigenetic dysregulation as a primary therapeutic target. Haematologica 2019;104(04):729-737

7 Sapienza MR, Fuligni F, Agostinelli C, et al; AIRC 5xMille consortium 'Genetics-driven targeted management of lymphoid malignancies and the Italian Registry on Blastic Plasmacytoid Dendritic Cell Neoplasm. Molecular profiling of blastic plasmacytoid dendritic cell neoplasm reveals a unique pattern and suggests selective sensitivity to NF-kB pathway inhibition. Leukemia 2014;28 (08):1606-1616

8 Demiröz AS, Demirkesen C, Salihoğlu A, Tüzüner N. Blastic plasmacytoid dendritic cell neoplasia: a single center experience. Turk J Haematol 2020;37(01):48-52

9 Bekkenk MW, Jansen PM, Meijer CJLM, Willemze R. CD56+ hematological neoplasms presenting in the skin: a retrospective analysis of 23 new cases and 130 cases from the literature. Ann Oncol 2004;15(07):1097-1108

10 Suzuki R, Nakamura S, Suzumiya J, et al; NK-cell Tumor Study Group. Blastic natural killer cell lymphoma/leukemia (CD56-positive blastic tumor): prognostication and categorization according to anatomic sites of involvement. Cancer 2005;104(05):1022-1031

11 Assaf C, Gellrich S, Whittaker S, et al. CD56-positive haematological neoplasms of the skin: a multicentre study of the Cutaneous Lymphoma Project Group of the European Organisation for Research and Treatment of Cancer. J Clin Pathol 2007;60(09): 981-989

12 Cota C, Vale E, Viana I, et al. Cutaneous manifestations of blastic plasmacytoid dendritic cell neoplasm-morphologic and phenotypic variability in a series of 33 patients. Am J Surg Pathol 2010; 34(01):75-87

13 Rauh MJ, Rahman F, Good D, et al. Blastic plasmacytoid dendritic cell neoplasm with leukemic presentation, lacking cutaneous involvement: case series and literature review. Leuk Res 2012; 36(01):81-86

14 Jegalian AG, Facchetti F, Jaffe ES. Plasmacytoid dendritic cells: physiologic roles and pathologic states. Adv Anat Pathol 2009;16 (06):392-404

15 Petrella T, Facchetti F. Tumoral aspects of plasmacytoid dendritic cells: what do we know in 2009? Autoimmunity 2010;43(03):210-214

16 Lucioni M, Novara F, Fiandrino G, et al. Twenty-one cases of blastic plasmacytoid dendritic cell neoplasm: focus on biallelic locus 9p21.3 deletion. Blood 2011;118(17):4591-4594

17 Shi Y, Wang E. Blastic plasmacytoid dendritic cell neoplasm: a clinicopathologic review. Arch Pathol Lab Med 2014;138(04):564-569

18 Swerdlow S, Campo E, Harris NL, et al. WHO Classification of Tumours of Haematopoietic and Lymphoid Tissues. 4th ed. Lyon, France: IARC; 2008

19 Arber DA, Orazi A, Hasserjian R, et al. The 2016 revision to the World Health Organization classification of myeloid neoplasms and acute leukemia. Blood 2016;127(20):2391-2405

20 Deotare U, Yee KWL, Le LW, et al. Blastic plasmacytoid dendritic cell neoplasm with leukemic presentation: 10-color flow cytometry diagnosis and hyperCVAD therapy. Am J Hematol 2016;91 (03):283-286 
21 Deotare U, Kim DD, Michelis FV, Lipton JH, Lipton JH. Allogeneic hematopoietic stem cell transplantions in blastic plasmacytoid dendritic cell neoplasm in first complete remission: an effective therapy for a rare disease. Leuk Lymphoma 2016;57(08):1942-1944

22 Gruson B, Vaida I, Merlusca L, et al. L-asparaginase with methotrexate and dexamethasone is an effective treatment combination in blastic plasmacytoid dendritic cell neoplasm. $\mathrm{Br} \mathrm{J}$ Haematol 2013;163(04):543-545

23 Gilis L, Lebras L, Bouafia-Sauvy F, et al. Sequential combination of high dose methotrexate and L-asparaginase followed by allogeneic transplant: a first-line strategy for CD4+/CD56+ hematodermic neoplasm. Leuk Lymphoma 2012;53(08):1633-1637

24 Reimer P, Rüdiger T, Kraemer D, et al. What is CD4+CD56+ malignancy and how should it be treated? Bone Marrow Transplant 2003;32(07):637-646

25 Leitenberger JJ, Berthelot CN, Polder KD, et al. CD4+ CD56+ hematodermic/plasmacytoid dendritic cell tumor with re- sponse to pralatrexate. J Am Acad Dermatol 2008;58(03): 480-484

26 DiNardo CD, Pratz K, Pullarkat V, et al. Venetoclax combined with decitabine or azacitidine in treatment-naive, elderly patients with acute myeloid leukemia. Blood 2019;133(01):7-17

27 Ulrickson ML, Puri A, Lindstrom S, Cassaday RD, De Padova N, Becker PS. Gemcitabine and docetaxel as a novel treatment regimen for blastic plasmacytoid dendritic cell neoplasm. Am J Hematol 2017;92(05):E75-E77

28 Khwaja R, Daly A, Wong M, Mahé E, Cerquozzi S, Owen C. Azacitidine in the treatment of blastic plasmacytoid dendritic cell neoplasm: a report of 3 cases. Leuk Lymphoma 2016;57(11): 2720-2722

29 Frankel AE, Woo JH, Ahn C, et al. Activity of SL-401, a targeted therapy directed to interleukin-3 receptor, in blastic plasmacytoid dendritic cell neoplasm patients. Blood 2014;124(03): 385-392 